\title{
Calculation of the Hardness of a Multi-Element Equiatomic Metal Alloy
}

\author{
Zakarian DA* and Khachatrian AV \\ Frantzevich Institute for Problems of Materials Science NAS Ukraine, Kiev
}

*Corresponding author: Zakarian DA, Frantzevich Institute for Problems of Materials Science NAS Ukraine, Kiev

\begin{abstract}
A phenomenological approach has been developed for the mathematical description of the hardening of high-entropy alloys (HEAs) and a quantitative assessment of their hardness. It is shown that in HEAs with a bcc lattice, the degree of hardening is always greater than in HEAs with a fcc lattice (with the same number of elements in alloys). To calculate the relative hardening of the alloy, the type of crystal lattice and its parameter value are required. The calculation results are consistent with experimental data. Basic parameters of HEAs are calculated from first principles. The proposed method can be considered acceptable for assessing the hardness and degree of hardening of multi-element alloys (HEAS).
\end{abstract}

Keywords: Multielement alloys; energy of interaction of elements; mismatch parameter; distortion density; hardness; heas

\section{Introduction}

One of the most common characteristics that determine the quality of metals and alloys, the possibility of their use in various designs and under various working conditions, is hardness. Hardness tests are performed more often than determining other mechanical characteristics of metals: strength, elongation, etc. A specific method for determining hardness is selected based on the properties of the material, measurement tasks, conditions for its implementation, available equipment, etc. Hardness is a technical characteristic of a material, and depends on a set of physical properties, such as: interatomic distances, coordination number, number of valence electrons, nature of the chemical bond, brittleness, elasticity, viscosity, etc. And calculation it from first principles without taking into account experimental data at the present stage is impossible, especially if this applies to multielement or high-entropy alloys (HEAs).

It is generally accepted that one of the fundamental concepts for a HEAs is that the lattice structure of a solid solution is greatly distorted. The displacement of atoms from their ideal positions is given as the reason for the manifestation of a number of observed physical and mechanical properties. It is believed that the distortion of the crystal lattice of the HEAs creates an obstacle to the free movement of dislocations, i.e. serves as a deterrent to plastic deformation. Therefore, to describe the hardening of the HEAs, the main emphasis in the research is placed on taking into account the difference in the radii of the atoms present in the alloy. Basically, the mean quadratic displacement of atoms from the equilibrium position in an ideal, undistorted lattice is used as a mismatch parameter [1,2].

In [1], it was found that the normalized strength at $0 \mathrm{~K}$ for $8 f c c$ alloys, five-element equiatomic HEA CrMnFeCoNi and its derivatives is proportional to the mean square displacement. Thus, arguing that the root mean square displacement are an important factor in predicting the yield strength of alloys at $0 \mathrm{~K}$. For the same alloys in [2], from the results of experimental studies it was found that not always an increase in distortion necessarily leads to an improvement in physical characteristics. Those. in assessing the mechanical characteristics of a HEAs, it is necessary to take into account not only the parameters of the mismatch of atomic radii, but also the values of the interaction potential of different elements. Here it is appropriate to cite the statement of the authors of [3], who believe that at present there is no clear evidence that the lattice distortions in the HEAs are much higher than in conventional alloys. Another method for assessing hardening in HEAs is solid solution hardening, where Fleischer, Lyabush and Gippen approaches are used, which are mainly used for binary alloys [4].

The vast majority of works are methods that work well on some HEAs and much worse on others. The mechanism of strengthening the HEAs is the most important feature of this class of materials and 
this issue, so far, has neither a clear explanation, nor fundamental confirmation. The main emphasis is on creating barriers to the movement of dislocations and, less commonly, on the features of the interaction of dissimilar atoms with each other. Undoubtedly, the asymmetric environment of each of the atoms in the crystal of a disordered alloy leads to the fact that the equilibrium state of the alloy is accompanied by the appearance of distortions of its crystal lattice. These distortions depend only on the composition of the alloy; they do not arise and do not disappear during the thermomechanical treatment. In this paper, we propose a "hybrid" method - a combination of first-principle methods, the use of experimental data (relating to pure metals) and a phenomenological approach to assess the hardness of a HEAs with a $b c c$ and $f c c$ singlephase structure.

\section{Calculation of Basic Parameters of a HEAs}

To calculate the basic parameters of a HEAs, an a priori pseudopotential is used [5].When studying the properties of transition metals using the pseudopotential method, the state of $d$ electrons is taken into account, which occupy an intermediate position between highly localized internal states and free electrons in the crystal. The interaction of $d$ electrons with conduction electrons leads to the appearance of two additional terms in the pseudopotential of the ion associated with the overlap of $s$ and $d$ waves and with the resonant interaction of $d$ waves. We consider the case when the system consists of different metal atoms with concentrations $c_{i} \sum_{i=1}^{N} c_{i}=1$ forming an alloy of the substitution type.

The pseudopotential of the alloy is determined according to the conditions of its additive nature ${ }^{\bar{V}(q)}=\sum_{i} C_{i}(q)$ where $v i(q)$ is the pseudopotential of the $\mathrm{i}^{\text {th }}$ ion.

Note that in high-entropy alloys, quantum-mechanical calculations are carried out in the same way as for pure metals, but with an average pseudopotential of the form [1]. The energy of the electron-ion system is calculated using the perturbation theory in pseudopotential up to second order inclusive. In order to evaluate the hardening of multi-element alloys by theoretical methods, it is necessary to study the mechanical behavior of an increasingly complicated sequence of alloys related in composition. Using an a priori pseudopotential [5], for pure metals and alloys with an $f c c$ and $b c c$ structure, the type of crystal structure, lattice parameters, and elastic moduli for the family of equiatomic nickel $\mathrm{CrMnFeCoNi}$ HEAs are determined from the minimum energy. The calculated data are consistent with experimental data [6,7] (Figure 1) Table 1.

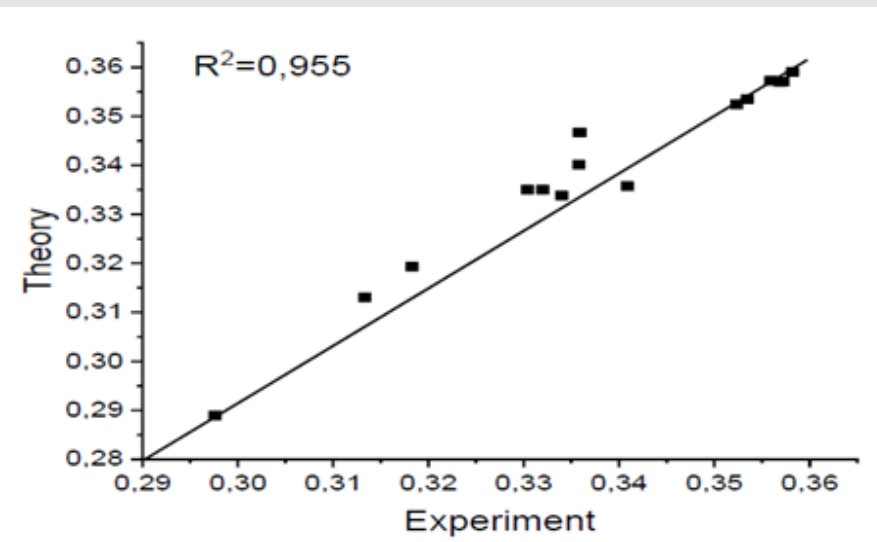

Figure 1: Lattice parameter of multi-element alloys with $b c c$ and $f c c$ lattices and their experimental values $(2,7,9)$. $R^{2}$ is the coefficient of determination

Table 1: The calculated $\left(\mathrm{E}_{\mathrm{c}}\right)$ and experimental $\left(\mathrm{E}_{\mathrm{ex}}\right)$ values of the elastic moduli of Ni based alloys (in $\left.\mathrm{GPa}\right)$.

\begin{tabular}{|c|c|c|c|c|c|c|c|}
\hline Material & CrMnFeCoNi & CrFeCoNi & FeCoNi & CrCoNi & CoNi & FeNi & Ni \\
\hline $\mathbf{E}_{\mathrm{c}}$ & 203,78 & 218,97 & 164,79 & 227,85 & 215,24 & 168,72 & 202,54 \\
\hline $\mathbf{E}_{\mathrm{ex}}[\mathbf{2}]$ & 201,6 & 215,04 & 162,0 & 226,2 & 216,7 & 166,16 & 199,12 \\
\hline
\end{tabular}

As for the mean square displacement, paired interatomic potentials are used to determine it. If we calculate the pair interactions for any two atoms in the alloy (i.e., surrounded by random types of atoms), then the result will be the same for all pairs of atoms, because the interatomic distance is determined using the average lattice parameter. Therefore, to determine the interatomic distance of different types of atoms, we carry out calculations for alloys consisting of two types of atoms. To determine the distances between atoms of the same type, calculations are carried out as for pure metals. Theoretically, it is possible to evaluate the influence of any new element on the characteristics of the selected element in the alloy. To assess the mechanical characteristics of the HEAs, averaged basic parameters are used, such as the lattice parameter, atomic radius, and root mean square displacement [8]. 


\section{Modeling the Structure and Properties of HEAs, Calculation Results and Discussion}

We model the structure of a HEA with $n$ elements. Depending on the type of crystal lattice, for a complete description of the structure of the HEAs, it is necessary to take into account:

a. The number of unit cells where pair interactions are realized both between the same type and the different type elements.

b. The number of unit cells, which takes into account the diversity of the population of atoms (taking into account the concentration ratio).

The number of obtained unit cells depends not only on the type of crystal lattice, but also on the number of elements. A set of these elementary cells can be represented as an "abstract, virtual particle" possessing the properties of a HEA and containing the basic elements of its structure. For example, in a three-element alloy ( $\mathrm{n}=3), 12$ unit cells are sufficient to describe the properties and structure, if the alloy has an $f c c$ lattice, and in the case of a bcc lattice, there are 6 cells in which atoms of different sorts are randomly distributed, exhausting all possible distribution options. The set of these unit cells is sufficient for calculating the basic parameters of the alloy from first principles. The totality of these unit cells carries information about the property and structure of the alloy. The selected group with a certain number of elementary cells of the HEA is sufficient for the mathematical description of the basic properties and structure of the alloy.

The volume of the "particle" can be estimated by the formula $\Omega_{x}=N \Omega$, where $N$ is the number of unit cells, the combination of which represents the "characteristic particle", $\Omega$ is the average volume of the unit cell of the alloy. The linear particle size will be $d=(N . \Omega)^{1 / 3}$.

We introduce the concept of distortion density, which is directly proportional to the distortion $\Delta r$ and inversely proportional to the linear size of the "particles" $\rho=\Delta r / d$. We use the main feature of the HEAs - the distortion of the crystal lattice, as a deterrent to plastic deformation and the movement of dislocations.

Our task is to use this feature of the HEAs without affecting the mechanism of movement of dislocations in the HEAs. Those, we are modeling not the process of inhibition of dislocation motion, but the consequence of this process is the hardening effect. An increase in the distortion density, i.e., a decrease in the effective size $d_{1}=\frac{1}{\rho}$, leads to an increase in the force that impedes the propagation of plastic deformation and to hardening of the material. In this context, a HEAs can be described as a "polycrystalline" material consisting of "particles" or "grains", at which "boundaries" inhibit the movement of linear defects and, accordingly, harden the material. In this case, the degree of distortion of the crystal lattice or "distortion density" acts as a boundary. In this regard, the strengthening of the HEAs can be estimated by analogy with the Hall-Petch law in the form:

$$
H=H_{0}\left(1+c / d_{1} \frac{1}{2}\right)=H_{0}\left(1+c(\Delta r / d) \frac{1}{2}\right)
$$

where $H_{0}=\Sigma C_{i} H_{i}$ is the average alloy hardness, estimated according to the rule of the mixture ( $\mathrm{Hi}$ is the hardness of a single crystal consisting of the $\dot{i}^{\text {th }}$ element included in the alloy), $\boldsymbol{c}$ is the material constant, which is proportional to the number of elements included in the alloy. As for distortion, it is possible to use different versions of its representation [6]. Based on the results of a computational experiment, the most unambiguous, therefore, successful option is to present the distortion in the form $\Delta r=r_{\max }-r_{\mathrm{mi}}$ $\left(r_{\max }\right.$ and $r_{\min }$ are the maximum and minimum atomic radii of the elements in the crystalline modification of the alloy). Given that the unit cell volume $\Omega=\mathrm{a}^{3}$ ( $a$ is the lattice parameter), we obtain:

$$
H=H_{0}\left(1+\frac{c .(\Delta r)^{1 / 2}}{(a)^{1 / 2} \cdot N^{1 / 6}}\right.
$$

With an increase in the number of types of atoms, the distortion of the crystal lattice increases. Using relation (3) for substitutional type alloys (NiCo) and comparing the calculated and experimental data on hardness, we find that $c=3.8 n$ ( $n$ is the number of elements in the alloy). For a relative increase in hardness, we obtain

$$
\Delta H / H_{0}=\left(H-H_{0}\right) / H_{0}=\frac{3,8 n}{N^{1 / 6}} \cdot\left(\frac{\Delta r}{a}\right)^{1 / 2}
$$

The relative increase in hardness depends on the ratio of the parameter $\Delta r$ to the crystal lattice parameter of the alloy $a$. If the first factor is related to the difference in atomic radii of the elements, then the second is indirectly related to the potentials of different elements, as well as their interaction. Formula (4) takes into account the effect on the mechanical characteristics of the distortion of the crystal lattice.

A computational experiment was carried out for a HEAs with an $f c c$ lattice, mainly for an equiatomic HEA Minecon and its derivatives with nickel, as well as 5 and 6 element equiatomic alloys with a $b c c$ lattice. All alloys have a stable single-phase structure. The average difference in the atomic radii of the elements (which are part of the alloy) from the average radius of the alloy is not more than $6.6 \%$. From the results obtained Table 2, it follows that using the relation (3), it is possible to evaluate the hardness of various multi-element, including two-element and three-element metal alloys. The experimental values of hardness taken from [9] for $f c c$ alloys are overestimated in comparison with the calculated values. But, as noted in [9], in these alloys, in addition to solid solutions of HEAs based on $f c c$ lattices, solid solutions based on $b c c$ lattices, as well as intermetallic compounds, can be present. In other cases, the results of the experiment and calculation are close. If we compare the hardness values of the equiatomic HEA CrFeCoNiMn and its derivatives with the presence of nickel with an elastic modulus Table 1, we see that there is a direct relationship between the elastic modulus and hardness for some alloys when the number of elements in the alloy is $n \geq 3$. As shown calculation results and experimental data [9] there is not always direct proportionality between the elastic modulus and hardness in both $b c c$ and $f c c$ HEAs.

We estimate the relative increase in hardness. Following relation (4), we present the relative increase in hardness $\Delta \mathrm{H} / \mathrm{H}_{0}$ of alloys as a function of the parameter $\rho_{n} \frac{n\left(\Delta \Delta / s^{1 / 2}\right.}{N^{1 / 6}}$ of $f c c$ and $b c c$ alloys in 
(Figure 2). The left part of the figure shows the results of calculating the increase in hardness as a function of the parameter $\rho_{n}$ for nickelbased alloys consisting of two, three, four and 5 elements having an $f c c$ structure. An increase in $\rho_{n}$ leads to an increase in hardness. The greatest effect of hardening of solid solutions from equiatomic HEA of $\mathrm{CrMnFeCoNi}$ and its derivatives with the presence of nickel with an $f c c$ structure is observed in a 5-element alloy (FeNiCoCrMn), slightly lower than a 4-element alloy (FeNiCoCr), when the first place in terms of hardness in this family NiCoCr. The right part of the figure shows the results of hardening for $b c c$ alloys. A comparison of the relative strengths of the HEA shows that HEAs with a bcc lattice are stronger than the alloys with an $f c c$ lattice, provided that the number of elements in the alloy is equal. This confirmation also

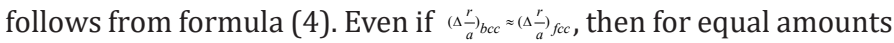
of elements in the two alloys, the coefficient $\left.{ }^{\left(1 / N^{1 / 6}\right.}\right)_{b c c}>\left(1 / N^{1 / 6}\right)_{f c c}$ which means that the distortions density is higher in the bcc HEAs than in the $f c c$.

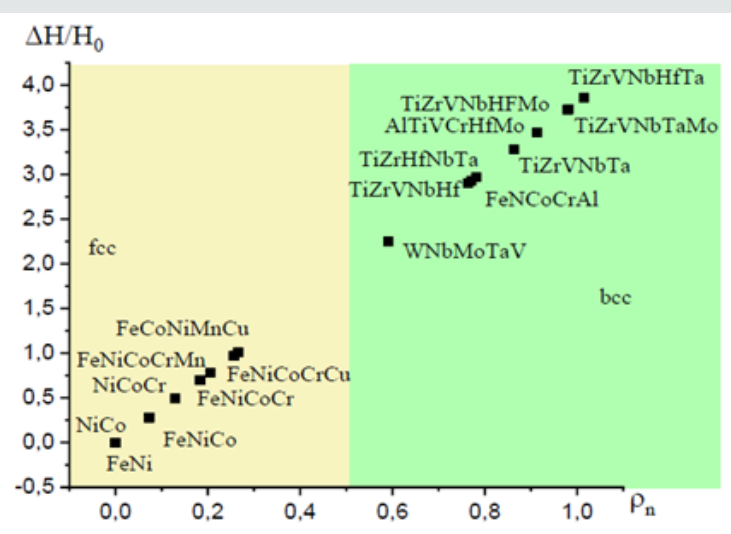

Figure 2: Relative hardening for multi-element alloys with bcc and fcc lattice

Table 2: The calculated values of the lattice parameter (a), the maximum difference in atomic radii $(\Delta \mathrm{r})$, the average value of the alloy hardness $(\mathrm{H} 0)$, the hardness for different metal alloys with bcc and fcc structure $(\mathrm{H})$ and their experimental values (Hexp).

\begin{tabular}{|c|c|c|c|c|c|c|}
\hline Material & $\mathbf{N}$ & $\mathrm{a}, \mathrm{nm}$ & $\Delta \mathbf{r}, \mathbf{n m}$ & $\mathrm{H}_{0}, \mathrm{GPa}$ & H, GPa & $\mathrm{H}_{\mathrm{exp}}, \mathrm{GPa}$ \\
\hline \multicolumn{6}{|c|}{$f c c$ structure } & [2] \\
\hline $\mathrm{Ni}$ & 1 & 0,35238 & 0 & 0,638 & 0,638 & 0,686 \\
\hline NiCo & 5 & 0,3535 & 0,0008 & 0,8405 & 1,073 & 1,047 \\
\hline $\mathrm{FeNi}$ & 5 & 0,3590 & 0,00081 & 0,623 & 0,795 & 0,863 \\
\hline FeNiCo & 12 & 0,3565 & 0,00152 & 0,763 & 1,138 & 0,973 \\
\hline $\mathrm{NiCoCr}$ & 12 & 0,3572 & 0,00306 & 0,91367 & 1,550 & 1,465 \\
\hline FeNiCoCr & 19 & 0,3561 & 0,00252 & 0,83725 & 1,492 & 1,180 \\
\hline CrFeCoNiMn & 31 & 0,3599 & 0,00296 & 0,701 & 1,383 & $2,4[9]$ \\
\hline $\mathrm{CrFeCoNiCu}$ & 31 & 0,3568 & 0,00316 & 0,743 & 1,495 & $2,7[9]$ \\
\hline FeCoNiMnCu & 31 & 0,3561 & 0,003159 & 0,6708 & 1,349 & $4,0[9]$ \\
\hline \multicolumn{6}{|c|}{ bcc structure } & [7-9] \\
\hline FeNiCoCrAl & 15 & 0,2890 & 0,01740 & 0,703 & 27,905 & \\
\hline TiZrVNbTa & 15 & 0,3351 & 0,02463 & 1,01 & 4,323 & 3,78 \\
\hline TiZrHfNbTa & 15 & 0,3357 & 0,01967 & 10,108 & 3,971 & 3,826 \\
\hline WNbMoTaV & 15 & 0,3197 & 0,01103 & 16,467 & 5,348 & 5,25 \\
\hline TiZrVNbHf & 15 & 0,3401 & 0,01957 & 1,125 & 4,390 & 4,1 \\
\hline TiZrNbVHfTa & 21 & 0,3467 & 0,02733 & 1,078 & 5,234 & 4,4 \\
\hline AlTiVCrNbMo & 21 & 0,3130 & 0,01637 & 1,043 & 4,669 & 5.1 \\
\hline TiZrVNbTaMo & 21 & 0,3338 & 0,02463 & 10,413 & 4,926 & 4,6 \\
\hline TiZrVNbHfMo & 21 & 0,3350 & 0,02460 & 11,897 & 5,617 & 5,16 \\
\hline
\end{tabular}


It is generally accepted that a model adequately describes a physical process if it obeys two conditions:

a. Physically justified.

b. The calculation results have experimental confirmation.

The proposed model satisfies these two conditions.

In all used methods (approaches), it is believed that the mechanical characteristics are directly proportional to distortions (mismatch of atomic radii of the elements). However, not all alloys obey this ratio. In our proposed model, in fact, physical characteristics are proportional to distortion to the degree of $1 / 2$. This approach allows to comprehensively take into account the features of the distorted crystal lattice of HEAs when assessing their physical and mechanical characteristics. It follows directly from relation (4) that for any two-element alloy of the substitution type, the relative hardening is always less than that of any threeelement single-phase alloy, even if $\Delta r$ of the two-element alloy is greater than that of the three-element one.

\section{Summary}

For hardening, the number of elements included in the composition of the alloy, the type of crystal lattice and the density of distortion are of key importance. The last parameter depends on both the mismatch parameter and the interatomic interaction potential. The role of the phenomenological approach is the mathematical description of the inhibition of plastic deformation. As a braking force in HEAs, the concept of "grain size" is used, which is defined using a set of unit cells, which is a carrier of informationthe properties and structure of a multi-element alloy. The proposed method, using experimental information on the hardness of metals, can be considered acceptable for assessing the hardness of multi-element alloys (HEAs). The resulting analytical formula is of practical importance, because makes it possible to evaluate the degree of hardening for any single-phase equiatomic HEA, if the experimental data of the alloy are known - the type of crystal lattice and its parameter.

\section{References}

1. Norihiko LO, Koretaka Y, Katsshi T, Haruyuki I, Easo PG (2016) Atomic displacement in the $\mathrm{Cr} \mathrm{Mn}$ Fe Co Ni high-entropy alloy-A scaling factor to predict solid solution strengthening. AIP Advances 6(2): 12008.

2. Wu Zhenggang (2014) Temperature and Alloying Effects on the Mechanical Properties of Equiatomic FCC. Solid Solution Alloys. University of Tennessee, Knoxville.

3. Lewis RO, Nicholas GJ (2018) Lattice distortions in high-entropy alloys. J Mater Res 33(19): 2946-2969.

4. Isaac Toda Caraballo, Pedro EJRDC (2015) Modelling solid solution hardening in high entropy alloys. Acta Materialia 85: 14-23.

5. Zakaryan DA, Kartuzov VV, Khachatryan AV (2015) Calculation of the basic physical and mechanical characteristics of highly entropic metal alloys. Mathematical models and computational experiment in materials science 17: S78-82.

6. Khachatryan AV (2018) Physical characteristics of equiatomic Nickel alloys and their relationship with distortions. Mathematical models and computational experiment in materials science 20: S115-120.

7. Firstov SA, Rogul TG, Krapivka NA, Ponomarev SS (2013) Effect of crystallization rate on the structure, phase composition and hardness of the high-entropy AlTiVCrNbMo alloy. Deformation and destruction of materials 10: C8-15.

8. Gorban VF, Krapivka NA, Firstov SA, Kurylenko DV (2018) The role of various parameters in the formation of the physico mechanical properties of high-entropy alloys with a bcc lattice. Physics of metals and materials science 119(5): 504-509.

9. Report III-6-16 (2018) NASU. Regularities of the formation of solid solutions with $b c c$ and $f c c$ lattice in high-centropium alloys and the influence of various factors on the phase, state, structure and physicomechanical properties.

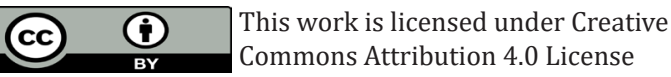

To Submit Your Article Click Here: $\quad$ Submit Article

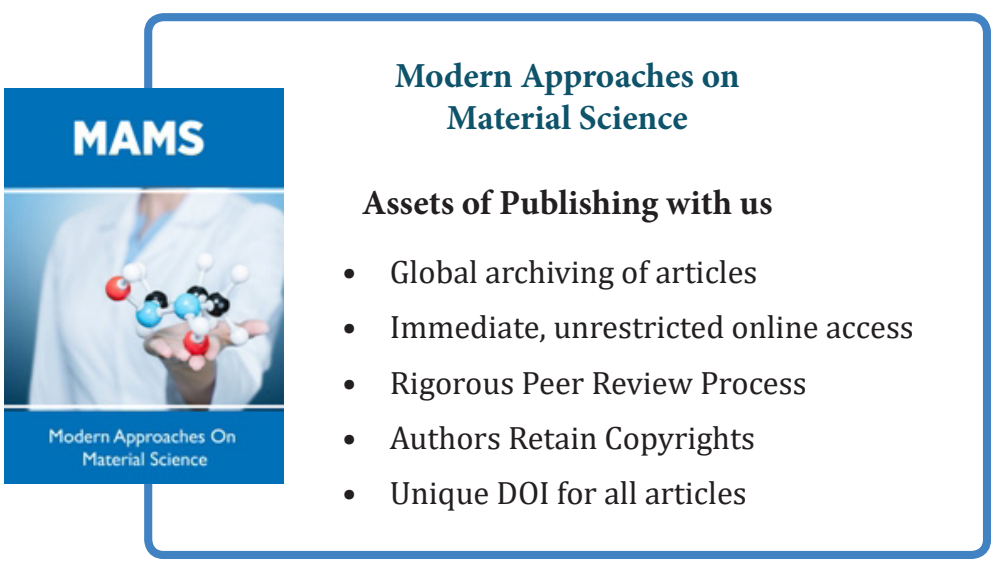

